\title{
A REJOINDER ON QUATERNIONIC PROJECTIVE REPRESENTATIONS
}

\author{
S.L. AdLER * AND G.G. EMCH ${ }^{\dagger}$ \\ * Institute for Advanced Study, Princeton, NJ 08540 \\ $\dagger$ Dept. of Mathematics, Univ. of Florida, Gainesville, FL 32611
}

\begin{abstract}
In a series of papers published in this Journal, a discussion was started on the significance of a new definition of projective representations in quaternionic Hilbert spaces. The present paper gives what we believe is a resolution of the semantic differences that had apparently tended to obscure the isssues.
\end{abstract}

We must first harmonize the notations in papers ${ }^{1,2,3,4}$ that were written more than thirty years apart, and for different audiences. Let $\mathcal{H}_{\mathbb{H}}$ be a quaternionic Hilbert space. In order to facilitate the transcription to Dirac's bra-ket notation, we write the multiplication by scalars on the right, with the scalar product defined to be linear in its second term:

$$
(\psi p, \phi q)=p^{*}(\psi, \phi) q
$$

in conformity with $|\phi q>=| \phi>q$.

Under the initial assumptions of Wigner ${ }^{5}$, reformulated by Bargmann ${ }^{6}$, or the assumptions of Emch and Piron ${ }^{7}$, a symmetry $\mu$ is defined as a map that preserves transition probabilities between rays, or equivalently as an automorphism of the ortho-complemented lattice $\mathcal{P}\left(\mathcal{H}_{\mathbb{H}}\right)$ the elements of which are the closed subspaces (i.e. the projectors) of the Hilbert space $\mathcal{H}_{\mathbb{H}}$.

The theorem known as Wigner's theorem (by physicists), and as the infinite dimensional version of the fundamental theorem ${ }^{8}$ of projective geometry (by mathematicians) asserts that every symmetry is implemented by a co-unitary operator $U$ satisfying :

$$
P \in \mathcal{P}\left(\mathcal{H}_{\mathbb{H}}\right) \quad \Rightarrow \quad \mu[P]=U^{*} P U
$$

with

$$
U(\psi q)=(U \psi) \alpha_{U}[q] \quad \forall \quad \psi \in \mathcal{H}_{\mathbb{H}} \quad \text { and } \quad q \in \mathbb{H}
$$

and

$$
\alpha_{U}[q]=\omega_{U}^{*} q \omega_{U} \quad \text { for some } \quad \omega_{U} \in \mathbb{H} \quad \text { with } \quad \omega_{U}^{*} \omega_{U}=1 \text {; }
$$

i.e. $\alpha_{U}$ is an automorphism of the field of quaternions. The co-unitarity of $U$ means that

$$
U^{*} U=U U^{*}=I \quad \text { so that } \quad(U \psi, U \phi)=\alpha_{U}[(\psi, \phi)]
$$


which reflects the fact that for a co-linear operator $A$ the adjoint is defined by

$$
\left(A^{*} \psi, \phi\right)=\alpha_{A}^{-1}[(\psi, A \phi)] .
$$

Conversely, every co-unitary operator implements a symmetry.

Finally, a symmetry determines the co-unitary operator that implements it, uniquely up to a "phase"; specifically the quaternionic form of Schur's lemma ${ }^{1}$ implies that two co-unitary operators $U_{1}$ and $U_{2}$ implement the same symmetry if and only if there exists a unit quaternion $\omega$, such that $U_{2}=U_{1} C_{\omega}$ where $C_{\omega}$ is the co-unitary operator defined by

$$
C_{\omega} \psi=\psi \omega
$$

Indeed,

$$
P \in \mathcal{P}\left(\mathcal{H}_{\mathbb{H}}\right) \quad \Rightarrow \quad C_{\omega}^{*} P C_{\omega}=P
$$

Hence for every symmetry separately, one can choose a unitary operator to implement this symmetry; and this unitary operator is unique up to a sign.

So far, and as long as each symmetry is treated separately, the above approach covers the premises of both Adler ${ }^{2}$ and Emch ${ }^{1}$.

When an abstract group $G$ is represented as a group of symmetries, i.e. when a symmetry $\mu(g)$ is assigned to every $g \in G$ in such a manner that

$$
\mu\left(g_{1}\right) \mu\left(g_{2}\right)=\mu\left(g_{1} g_{2}\right) \quad \forall \quad\left(g_{1}, g_{2}\right) \in G \times G,
$$

i.e.

$$
P \in \mathcal{P}\left(\mathcal{H}_{\mathbb{H}}\right) \quad \Rightarrow \quad \mu\left(g_{1}\right)\left[\mu\left(g_{2}\right)[P]\right]=\mu\left(g_{1} g_{2}\right)[P] \quad \forall \quad\left(g_{1}, g_{2}\right) \in G \times G,
$$

one can repeat the above procedure for each $g$ separately, and obtain a lifting by unitary operators $U(g)$ satisfying

$$
U\left(g_{1}\right) U\left(g_{2}\right)= \pm U\left(g_{1} g_{2}\right) .
$$

When $G$ is a Lie group, and $\mu$ is a continuous representation, the brutal lifting just described may, however, not lead to a continuous unitary representation. As physics needs continuity to define the observables corresponding to the generators of the unitary representation, it is reassuring to know that continuity obtains nevertheless ${ }^{1}$, as a result of the following procedure.

Firstly, one shows that there always exists a continuous local lifting by co-unitary operators, satisfying thus the condition

$$
U\left(g_{1}\right) U\left(g_{2}\right)=U\left(g_{1} g_{2}\right) C_{\omega\left(g_{1}, g_{2}\right)} .
$$

In this expression $C_{\omega}$ is a co-unitary operator, defined as in (5), where now $\omega=$ $\omega(.,$.$) is a continuous function of each of its arguments, takes its values in the$ unit quaternions, and satisfies, besides the trivial conditions $\omega(g, e)=\omega(e, g)$, the 2-cocycle condition:

$$
\omega\left(g_{1}, g_{2} g_{3}\right) \omega\left(g_{2}, g_{3}\right)=\omega\left(g_{1} g_{2}, g_{3}\right) \alpha_{U_{g_{3}}^{-1}}\left[\omega\left(g_{1}, g_{2}\right)\right] ;
$$


for the purpose of ulterior comparison with (13), we rewrite (10a) as:

$$
C_{\omega\left(g_{1}, g_{2} g_{3}\right)} C_{\omega\left(g_{2}, g_{3}\right)}=C_{\omega\left(g_{1} g_{2}, g_{3}\right)} U_{g_{3}}^{-1} C_{\omega\left(g_{1}, g_{2}\right)} U_{g_{3}}
$$

Secondly, one shows that such a lifting is always equivalent to a continuous, unitary, local, but true representation (i.e. no $\omega$, not even a \pm sign, ambiguity).

Thirdly, whenever the Lie group $G$ is simply connected, this can be extended to a continuous, unitary representation of the whole group $G$. In cases where the group is doubly connected (e.g. the rotation group in three dimensions), one only obtains the above result for its covering group; it is when one has to consider the group itself, that the \pm ambiguity of (8) can possibly manifest itself. As the latter amendment (covering multiply connected groups) is not germane to the issue on which we want to concentrate in this paper, we will not pursue that part of the discussion here.

The straightforward generalization we just sketched, extending to quaternionic Hilbert spaces the analysis familiar from the complex Hilbert spaces situation, presents one remarkable feature: the "phase reduction" is always locally trivial. Mathematically, this can be understood ${ }^{1}$ from the fact that the local phase reduction amounts to finding, up to equivalence, all the extensions ${ }^{9}$ of the Lie algebra of $G$ by the Lie algebra of the group of automorphisms of the field of quaternions; as the latter happens to be the semi-simple Lie algebra $s u(2, C)$, all such extensions are trivial ${ }^{10}$. In this respect the complex case is much more involved, as shown by Bargmann ${ }^{11}$. In particular, the phase reduction is not locally trivial for the Galilei group, a fact that is interpreted as viewing the mass as parametrizing the sectors of a superselection rule. Two attitudes are possible in this juncture. The first, which was chosen by Emch ${ }^{1}$, was to accept that Galilean QM is different in its quaternionic realization from what it is in its complex realization. The second is to pursue the issue, and to generalize the definition of a projective representation; this was recently proposed by Adler ${ }^{2}$.

Tranlated in the notation of this paper, Adler's proposal ${ }^{2}$ is to replace condition (9) by the weakened condition

$$
U\left(g_{1}\right) U\left(g_{2}\right)=U\left(g_{1} g_{2}\right) L_{\Omega\left(g_{1}, g_{2}\right)}
$$

where $L_{\Omega\left(g_{1}, g_{2}\right)}$ is the linear operator

$$
L_{\Omega\left(g_{1}, g_{2}\right)} \psi=\sum_{k} \phi_{k} \omega_{k}\left(g_{1}, g_{2}\right)\left(\phi_{k}, \psi\right) \quad \text { with } \quad \omega_{k}\left(g_{1}, g_{2}\right)^{*} \omega_{k}\left(g_{1}, g_{2}\right)=1
$$

and $\Phi=\left\{\phi_{k} \mid k=1,2, \ldots\right\}$ is a complete orthonormal basis in $\mathcal{H}_{\mathbb{H}}$, the same for all pairs $\left(g_{1}, g_{2}\right)$ of elements of $G$. Note that

$$
L_{\Omega\left(g_{1}, g_{2} g_{3}\right)} L_{\Omega\left(g_{2}, g_{3}\right)}=L_{\Omega\left(g_{1} g_{2}, g_{3}\right)} U_{g_{3}}^{-1} L_{\Omega\left(g_{1}, g_{2}\right)} U_{g_{3}} .
$$

While $\{11,13\}$ looks somewhat similar to $\{9,10 \mathrm{~b}\}$, there are major differences between these two formulations; our purpose in this paper is to delineate sharply the scope and reach of these variations.

Firstly, (9) is a direct consequence of the condition (7). Hence one should expect condition (7) to be violated by (11). This is indeed the case: see (16) below. Recall that (7) is the defining condition for the usual definition of a projective 
representation, as $\mathcal{P}\left(\mathcal{H}_{\mathbb{H}}\right)$ is the projective space associated to the vector space $\mathcal{H}_{\mathbb{H}}$. It is in fact equivalent to (9), and it is the condition Adler ${ }^{2}$ refers to as the defining property of a strong projective representation, in opposition to (11), which is equivalent to (16), and which he introduces as the definition of a weak projective representation.

Secondly, (9) is a relation among essentially co-unitary operators. It is true, as we just mentioned, that a powerful theorem ${ }^{1}$ allows to reduce the phases and thus to obtain a locally trivial continuous unitary representation, so that (9) becomes ultimately a relation between linear operators. Nevertheless, this reduction is not instructive in the present juncture since it is (9) itself [not (8)] that serves as a motivation for the extension (11). By contrast, (11) is in its very essence a relation between unitary operators; in particular $L$ is a linear operator (in fact a unitary operator) that involves the choice of a complete orthonormal basis $\Phi=\left\{\phi_{k} \mid k=\right.$ $1,2, \ldots\}$; i.e the focusing on one complete set of commuting observables, or more precisely, on a discrete, maximal abelian, real subalgebra

$$
\mathcal{A}_{\Phi}=\left\{A: \psi \in \mathcal{H}_{\mathbb{H}} \mapsto A \psi=\sum_{k} \phi_{k} a_{k}\left(\phi_{k}, \psi\right) \in \mathcal{H}_{\mathbb{H}}\right\}
$$

the minimal projectors of which are the projectors $P_{\phi_{k}}$ on the one-dimensional rays corresponding to each element $\phi_{k}$ of the chosen basis $\Phi$. We denote by $\mathcal{P}\left(\mathcal{A}_{\Phi}\right)$ the Boolean sublattice of $\mathcal{P}\left(\mathcal{H}_{\mathbb{H}}\right)$ generated by these projectors.

Thirdly, as a consequence of the above remark, whereas the co-linear operators $C_{\omega\left(g_{1}, g_{2}\right)}$ in (9) implement the trivial symmetry (see 6) - and are in particular independent of any choice of a Hilbert space basis - that is not the case for the symmetry implemented by the linear operators $L_{\Omega\left(g_{1}, g_{2}\right)}$. Indeed, we have generically only:

$$
P \in \mathcal{P}\left(\mathcal{A}_{\Phi}\right) \quad \Rightarrow \quad L_{\Omega\left(g_{1}, g_{2}\right)}^{*} P L_{\Omega\left(g_{1}, g_{2}\right)}=P
$$

Hence, the symmetry implemented by $U\left(g_{1} g_{2}\right)$ coincides with the symmetry implemented by $U\left(g_{1}\right) U\left(g_{2}\right)$ only for the elements of the distinguished maximal abelian algebra $\mathcal{A}_{\Phi}$ chosen to define the linear operators $L_{\Omega\left(g_{1}, g_{2}\right)}$ :

$$
P \in \mathcal{P}\left(\mathcal{A}_{\Phi}\right) \quad \Rightarrow \quad \mu\left(g_{1}\right)\left[\mu\left(g_{2}\right)[P]\right]=\mu\left(g_{1} g_{2}\right)[P] \quad \forall \quad\left(g_{1}, g_{2}\right) \in G \times G \quad
$$

This, compared to (7), is the major difference between the conditions defining weak vs strong projective representations. While both require, for each symmetry separately, that $\mu(g)$ be an automorphism of the whole system (a condition necessary to support the use of Wigner's theorem), the difference appears when it comes to the representation of a group of symmetries: the strong definition requires (7b), i.e. that $\mu$ is a representation on the full $\mathcal{P}\left(\mathcal{H}_{\mathbb{H}}\right)$ whereas the weak definition requires only (16), i.e. that this condition hold on $\mathcal{P}\left(\mathcal{A}_{\Phi}\right)$.

This is the price one must be prepared to pay for the relaxing from the "strong" condition (9) to the "weak" condition (11) - which is the generalization proposed by Adler ${ }^{2}$. At this price, it has become possible ${ }^{12,4,13}$ : to classify the irreducible weakly projective representations of connected Lie groups; to embed complex projective representations into weakly projective quaternionic representations (even when the Bargmann complex phase reduction is not locally trivial); to construct 
quaternionic coherent states (including the weakly projective case); and to discuss how, in the complex case, the weak condition (11) already implies the stronger condition of $(9)$.

After comparing their original motivations, the authors realized how they both had hoped to take advantage of the $S U(2)$ symmetry of the quaternions: Emch ${ }^{1}$ was interested in finding some natural coupling between the inhomogenous Lorentz group of special relativity and the internal symmetries then known in elementary particle theory; Adler ${ }^{2}$ was similarly interested in finding a source in the ray structure of Hilbert space for the color symmetry. It seems fair to say that, even with the generalization proposed by Adler ${ }^{2}$, the structure of the current quaternionic models for quantum theories is not (yet) rich enough to accomodate dreams that extend beyond the complex Hilbert space formalism.

\section{Acknowledgements}

The authors thank Dr. A. Jadczyk for discussions on matters related to this paper. The work of S.L. Adler was supported in part by the Department of Energy under Grant \#DE-FG02-90ER40542.

\section{REFERENCES}

1. G.G. Emch, Mécanique quantique quaternionienne et Relativité restreinte. I., Helv. Phys. Acta 36 (1963), 739-769; II., Helv. Phys. Acta 36 (1963), 770-788; Representations of the Lorentz Group in Quaternionic Quantum Mechanics (presented at the Lorentz Group Symposium, Summer 1964, Lectures in Theoretical Physics, Vol. VIIa, W.E. Brittin, ed., U.Colorado Press,, Boulder CO, 1964, pp. 1-36.

2. S.L. Adler, Projective Group Representations, J. Math. Phys. 37 (1996), 2352-2360; Quaternionic Quantum Mechanics and Quantum Fields, Oxford U.P., New York, 1995.

3. G.G. Emch, Comments on a Recent Paper by S.L. Adler ..., J. Math. Phys. 37 (1996), 6582-6585.

4. S.L. Adler, Response to the Comments by G. Emch ..., J. Math. Phys. 37 (1996), 6586-6589.

5. E.P. Wigner, Gruppentheorie, Vieweg \& Sohn, Braunschweig, Germany, 1931; Group Theory, Academic Press, New York, 1959.

6. V. Bargmann, Note on Wigner's Theorem on Symmetry Operations, J. Math. Phys. 5 (1964), 862-868.

7. G. Emch and C. Piron, Note sur les symétries en théorie quantique, Helv. Phys. Acta 35 (1962), 542-543; Symmetry in Quantum Theory, J. Math. Phys. 4 (1963), 469-473.

8. E. Artin, Geometric Algebra, Interscience, New York, 1957.

9. H. Cartan and S. Eilenberg, Homological Algebra, Princeton U.P., Princeton NJ, 1956.

10. N. Bourbaki, Eléments de mathématiques, Groupes et algèbres de Lie, Fasc. XXVI, Hermann, Paris, 1960.

11. V. Bargmann, On Unitary Ray Representations of Continuous Groups, Ann. of Math. 59 (1954), 1-46.

12. T. Tao and A.C. Millard, On the Structure of Projective Group Representations in Quaternionic Hilbert Space, J. Math. Phys. 37 (1996), 5848-5857.

13. S.L. Adler and A.C. Millard, Coherent States in Quaternionic Quantum Mechanics, J. Math. Phys. (to appear). 